Article

\title{
Fractional Lotka-Volterra-Type Cooperation Models: Impulsive Control on Their Stability Behavior
}

\author{
Rohisha Tuladhar ${ }^{1,+}$, Fidel Santamaria ${ }^{1,+}$ (D) and Ivanka Stamova ${ }^{2, *,+}$ \\ 1 Department of Biology, University of Texas at San Antonio, San Antonio, TX 78249, USA; \\ rohisha.tuladhar@utsa.edu (R.T.); fidel.santamaria@utsa.edu (F.S.) \\ 2 Department of Mathematics, University of Texas at San Antonio, San Antonio, TX 78249, USA \\ * Correspondence: ivanka.stamova@utsa.edu \\ + These authors contributed equally to this work.
}

Received: 1 August 2020; Accepted: 27 August 2020; Published: 31 August 2020

\begin{abstract}
We present a biological fractional $n$-species delayed cooperation model of Lotka-Volterra type. The considered fractional derivatives are in the Caputo sense. Impulsive control strategies are applied for several stability properties of the states, namely Mittag-Leffler stability, practical stability and stability with respect to sets. The proposed results extend the existing stability results for integer-order $n$-species delayed Lotka-Volterra cooperation models to the fractional-order case under impulsive control.
\end{abstract}

Keywords: biological Lotka-Volterra cooperation networks; fractional derivatives; impulsive control; Mittag-Leffler stability; practical stability; stability of sets

\section{Introduction}

The dynamics of Lotka-Volterra and related systems are traditionally applied to problems in population dynamics [1-4]. For example, the work in Reference [5] studied a delayed cooperation ecosystem composed of a number (n) of species using the following Lotka-Volterra model:

$$
\dot{x}_{i}(t)=r_{i}(t) x_{i}(t)\left[1-\frac{x_{i}\left(t-\tau_{i i}\right)}{a_{i}(t)+\sum_{\substack{j=1 \\ j \neq i}}^{n} b_{j}(t) x_{j}\left(t-\tau_{i j}\right)}-c_{i}(t) x_{i}\left(t-\tau_{i j}\right)\right],
$$

where $i, j=1, \ldots, n ; t \geq 0, x_{i}(t)$ represents the density of the species $i$ at time $t, r_{i}(t)$ are the intrinsic growth rates at time $t, a_{i}(t), b_{i}(t)$ and $c_{i}(t)$ are the cooperative coefficients, $\tau_{i i}$ denote the time delay due to gestation period of species $i, \tau_{i j}, j \neq i$ denote time delays related to the maturation of the species $i$ and $j$, respectively, $0 \leq \tau_{i j} \leq \tau, \tau=$ const,$(i=1,2, \ldots, n)$. It is assumed that only adult individuals of species $i$ can benefit the species $j$ and vice versa.

The model (1) has been originally introduced in Reference [6] for $n=2$ and $\tau_{i j}=0, i, j=1,2$, to describe the cooperative interaction between two species. Since then, it has been developed by several authors. See, for example, References [7-9].

An important objective in understanding biological systems is to determine their approach to equilibrium. Relative entropy, part of an information-theoretic approach, can be used to determine approach to equilibrium in biological systems, including the Lotka-Volterra type [10]. In addition, entropy methods are increasingly applied to biological models as robustness markers [11], as well as to determine information content in artificial neural networks [12].

Research on impulsive Lotka-Volterra and related systems has also increased because of their application to the study of population densities that are affected by some impulsive factors that 
cannot be ignored. Impulsive control strategies are also widely used in control theory of biological models [13-20]. In Reference [21] the author extended model (1) to the impulsive case and proved that the population dynamics of the system can be impulsively controlled. Furthermore, appropriate exterior or interior short-term changes in population densities may be used as controls. In general, impulsive controllers and impulsive control techniques demonstrate their efficiency in stability and synchronization of numerous applied models [22-24].

Fractional-order derivatives are increasingly being used to better reflect long-term dependence properties in biological systems [25-28]. Fractional Lotka-Voltera-type models have also been recently developed [29-34]. Indeed, the use of fractional derivatives and integrals has proven advantages in many applications across engineering and sciences [35-37]. In addition, the extended impulsive fractional-order case has also attracted the attention of many researchers [38-40]. Impulsive effects in fractional biological models are considered by several authors [38,39,41-43], including some fractional Lotka-Volterra models [39,44]. However, most of the studies considered competitive models, and to the best of our knowledge, there are no results on fractional cooperative models.

The asymptotic stability of equilibrium points is one of the most important tasks in the study of biological network systems. Asymptotic stability theory has been applied to a wide range of integer-order Lotka-Volterra systems to explain the behavior of the system's solutions using stabilization control law [1-3,5,7-9,21,23]. For fractional-order models the concept of Mittag-Leffler stability, introduced in Reference [45], is adapted as a generalization of the exponential stability notion in integer-order systems [46-48]. Very recently, the Mittag-Leffler notion has been also applied to fractional-order control problems [49-51], including impulsive controls in neural network systems [47,52-54]. The Mittag-Leffler stability and impulsive control strategies have not been investigated for fractional generalizations of the cooperative Lotka-Volterra model (1) which are important and challenging problems and one of the main goals of the present research.

The concepts of practical stability [55,56] and stability of sets [57] have been applied to fractional-order systems [58-61]. Using a practical stability approaches is beneficial in the cases when the equilibrium of the considered system is unstable in the classical Lyapunov or Mittag-Leffler sense, but its performance may be sufficient for a particular application [56]. Stability of sets generalizes the concept of stability of a single solution and is suitable to numerous real-world applications $[62,63]$. However, due to the complexity of the problem, no results on practical stability and stability of sets properties have been reported so far for fractional Lotka-Volterra-type models.

Stimulated by the above discussion, in this paper, we study impulsive control strategies for the Mittag-Leffler stability, practical stability, and stability of sets behavior of the fractional-order modification of the $n$-species delayed cooperation Lotka-Volterra type model (1). Caputo's fractional-order approach is adopted in our research. In Section 2 we will first introduce the fractional generalization of the biological network model (1). Some definitions and lemmas are also presented. Section 3 is devoted to our Mittag-Leffler stability results via impulsive controllers. In Section 4 the practical stability results for the fractional impulsive control biological model are derived. Section 5 generalizes the results in Sections 3 and 4, and offers stability of sets results. In Section 6 examples and simulations are given. Section 7 presents our conclusions and future directions.

\section{Fractional Lotka-Volterra-Type Model and Preliminary Notes}

We will use the following notations: $\mathbb{R}_{+}=[0, \infty), \mathbb{R}^{n}$ is the $n$-dimensional Euclidean space and $\|x\|=\left|x_{1}\right|+\ldots+\left|x_{n}\right|$ denote the norm of $x \in R^{n}$.

Let $u \in C^{1}[[0, b], \mathbb{R}], b>0$. For any $0<q<1$, following Reference [36], we consider the Caputo fractional derivative of order $q$ with the lower limit 0 for a function $u$, defined as

$$
{ }^{C} D_{t}^{q} u(t)=\frac{1}{\Gamma(1-q)} \int_{0}^{t} \frac{u^{\prime}(\sigma)}{(t-\sigma)^{q}} d \sigma, t \geq 0,
$$


where $\Gamma$ is the Gamma function and $u^{\prime}$ is the first-order ordinary derivative of the function $u$.

Since introducing fractional-order derivatives in population dynamics models has important practical significance and research values [25-34], we generalize the model (1), and introduce the following model with fractional-order derivatives

$$
{ }^{C} D_{t}^{q} x_{i}(t)=r_{i}(t) x_{i}(t)\left[1-\frac{x_{i}\left(t-\tau_{i i}\right)}{a_{i}(t)+\sum_{\substack{j=1 \\ j \neq i}}^{n} b_{j}(t) x_{j}\left(t-\tau_{i j}\right)}-c_{i}(t) x_{i}\left(t-\tau_{i j}\right)\right],
$$

where $i=1,2, \ldots, n, n \geq 2,0<q<1$, the functions $r_{i}(t), a_{i}(t), b_{i}(t)$ and $c_{i}(t)$ are continuous, positive and bounded on $\mathbb{R}_{+}$.

The fractional derivatives in the Lotka-Volterra-type cooperation model (2) will contribute for a better description of the population interactions characteristics and will improve the degree of freedom in comparison with the model (1) [35,36].

We will consider the following initial condition

$$
x_{i}\left(s ; 0, \varphi_{0}\right)=\varphi_{i 0}(s), s \in[-\tau, 0]
$$

associated with the model (2), where the initial function $\varphi_{0}:[-\tau, 0] \rightarrow \mathbb{R}^{n}, \varphi_{0}=\left(\varphi_{10}, \varphi_{20}, \ldots, \varphi_{n 0}\right)^{T}$ is continuous, positive and bounded on $[-\tau, 0]$.

For a continuous function $g(t)$ defined on $[0, \infty)$, we denote

$$
g^{L}=\inf _{t \in[0, \infty)} g(t), g^{M}=\sup _{t \in[0, \infty)} g(t) .
$$

The goal of our research is to investigate the effects of impulsive external perturbations on the stability behavior of the model (2) at some fixed points $t_{1}, t_{2}, \ldots$ such that

$$
0<t_{1}<t_{2}<\ldots<t_{k}<\ldots
$$

and $\lim _{k \rightarrow \infty} t_{k}=\infty$. To this end we will add impulsive controllers that can be used to synchronize the densities of all species onto that of system (2), and introduce the following impulsive control system

$$
{ }^{C} D_{t}^{q} y_{i}(t)=r_{i}(t) y_{i}(t)\left[1-\frac{y_{i}\left(t-\tau_{i i}\right)}{a_{i}(t)+\sum_{\substack{j=1 \\ j \neq i}}^{n} b_{j}(t) y_{j}\left(t-\tau_{i j}\right)}-c_{i}(t) y_{i}\left(t-\tau_{i j}\right)\right]+\eta_{i}\left(y_{i}(t)\right),
$$

where

$$
\eta_{i}\left(y_{i}(t)\right)=\sum_{k=1}^{\infty} I_{i k}\left(y_{i}(t)\right) \delta\left(t-t_{k}\right)
$$

are the control inputs, $\delta(t)$ is the Dirac impulsive function, $y_{i}: \mathbb{R}_{+} \rightarrow \mathbb{R}_{+}, I_{i k}: \mathbb{R}_{+} \rightarrow \mathbb{R}, i=1, \ldots, n$, $k=1,2, \ldots$

Due to the incorporation of the Delta impulsive function the controller $\eta(t)$ has an effect on sudden changes in the states of the model (4) at the time instants $t_{k}$, that is, $\eta(t)$ is an impulsive control of (4). For $t>0$ and $i=1,2, \ldots, n$, we denote

$$
f_{i}\left(t, y_{i}(t)\right)=r_{i}(t) y_{i}(t)\left[1-\frac{y_{i}\left(t-\tau_{i i}\right)}{a_{i}(t)+\sum_{\substack{j=1 \\ j \neq i}}^{n} b_{j}(t) y_{j}\left(t-\tau_{i j}\right)}-c_{i}(t) y_{i}\left(t-\tau_{i j}\right)\right] .
$$


From (4), we have that $[35,36,39,61], \eta_{i}\left(y_{i}(t)\right)=0$ for $t \neq t_{k}, k=1,2, \ldots ., i=1,2, \ldots, n$, and

$$
\begin{gathered}
y_{i}\left(t_{k}+h\right)-y_{i}\left(t_{k}\right)=\frac{1}{\Gamma(q)} \int_{0}^{t_{k}+h}\left(t_{k}+h-\sigma\right)^{q-1}\left[f_{i}\left(\sigma, y_{i}(\sigma)\right)+\eta_{i}\left(y_{i}(\sigma)\right)\right] d \sigma \\
-\frac{1}{\Gamma(q)} \int_{0}^{t_{k}}\left(t_{k}-\sigma\right)^{q-1}\left[f_{i}\left(\sigma, y_{i}(\sigma)\right)+\eta_{i}\left(y_{i}(\sigma)\right)\right] d \sigma \\
=\frac{1}{\Gamma(q)} \int_{t_{k}}^{t_{k}+h}\left(t_{k}+h-\sigma\right)^{q-1}\left[f_{i}\left(\sigma, y_{i}(\sigma)\right)+\eta_{i}\left(y_{i}(\sigma)\right)\right] d \sigma \\
+\frac{1}{\Gamma(q)} \int_{0}^{t_{k}}\left(\left(t_{k}+h-\sigma\right)^{q-1}-\left(t_{k}-\sigma\right)^{q-1}\right)\left[f_{i}\left(\sigma, y_{i}(\sigma)\right)+\eta_{i}(\sigma)\right] d \sigma
\end{gathered}
$$

where $h>0$ is sufficiently small. As $h \rightarrow 0^{+}$, we obtain

$$
\Delta y_{i}\left(t_{k}\right)=y_{i}\left(t_{k}^{+}\right)-y_{i}\left(t_{k}\right)=I_{i k}\left(y_{i}\left(t_{k}\right)\right),
$$

where $y_{i}\left(t_{k}^{+}\right)=\lim _{h \rightarrow 0^{+}} y_{i}\left(t_{k}+h\right)$.

Therefore, the correspondent $n$-dimensional fractional-order driven system is given by

$$
\left\{\begin{array}{l}
{ }^{c} D_{t}^{q} y_{i}(t)=y_{i}(t) r_{i}(t)\left[1-\frac{y_{i}\left(t-\tau_{i i}\right)}{a_{i}(t)+\sum_{\substack{j=1 \\
j \neq i}}^{n} b_{j}(t) y_{j}\left(t-\tau_{i j}\right)}-c_{i}(t) y_{i}\left(t-\tau_{i j}\right)\right], t \neq t_{k}, \\
y_{i}\left(t_{k}^{+}\right)=y_{i}\left(t_{k}\right)+I_{i k}\left(y_{i}\left(t_{k}\right)\right), i=1, \ldots, n, k=1,2, \ldots,
\end{array}\right.
$$

where the quantities $y_{i}\left(t_{k}\right)$ and $y_{i}\left(t_{k}^{+}\right)=\lim _{h \rightarrow 0^{+}} y_{i}\left(t_{k}+h\right)$ represent, respectively, the population densities of species $i$ before and after an impulsive perturbation at the moment $t_{k}$ and the continuous in $\mathbb{R}_{+}$functions $I_{i k}$ characterize the impact of the impulse effect on the species $i$ at the moments $t_{k}$.

For more results on fractional-order models under impulsive control where the Dirac delta function is incorporated in a similar sense, we refer the reader to References $[39,61]$ and the references therein.

Let $\phi_{0}:[-\tau, 0] \rightarrow \mathbb{R}^{n}, \phi_{0}=\left(\phi_{10}, \phi_{20}, \ldots, \phi_{n 0}\right)^{T}$ be a piecewise continuous function with points of discontinuity on $[-\tau, 0]$ at which it is continuous from the left. The class of all such functions will be denoted by $\mathcal{P C}$, and by $P C B\left[[-\tau, 0], \mathbb{R}^{n}\right]$ we will denote the class of all bounded functions $\phi_{0} \in \mathcal{P C}$. We denote by $y(t)=y\left(t ; 0, \phi_{0}\right)=\left(y_{1}\left(t ; 0, \phi_{0}\right), y_{2}\left(t ; 0, \phi_{0}\right), \ldots, y_{n}\left(t ; 0, \phi_{0}\right)\right)^{T}$ the solution of system (5), satisfying the initial conditions

$$
\left\{\begin{array}{l}
y_{i}\left(s ; 0, \phi_{0}\right)=\phi_{i 0}(s), s \in[-\tau, 0] \\
y_{i}\left(0^{+} ; 0, \phi_{0}\right)=\phi_{i 0}(0), i=1, \ldots, n .
\end{array}\right.
$$

Furthermore, from the biological point of view, we will restrict our attention only to the nonnegative solutions of the control system (5), and we will assume that if $\phi_{i 0}(s) \geq 0$, sup $\phi_{i 0}(s)<\infty$, $s \in[-\tau, 0], \phi_{i 0}(0)>0,1 \leq i \leq n$, then $y_{i}(t) \geq 0,1 \leq i \leq n, t \in[0, \infty)$. We also assume that $y_{i}+I_{i k}\left(y_{i}\right) \geq 0$ for $y_{i} \in \mathbb{R}_{+}, i=1,2, \ldots, n, k=1,2, \ldots$.

For some results on positive solutions of fractional Lotka-Volterra-type models we refer to References [32,33].

In addition, we assume that, if the functions $I_{i k}$ are such that $-y_{i} \leq I_{i k}\left(y_{i}\right) \leq 0$ for $y_{i} \in \mathbb{R}_{+}$, $i=1,2, \ldots, n, k=1,2, \ldots$, then there exist positive constants $m$ and $M<\infty$ such that

$$
m \leq y_{i}(t) \leq M, \quad t \in[0, \infty) .
$$


For integer-order Lotka-Volterra models with and without impulses, similar results can be found in References $[1,5,7-9,21,23]$. Since one of the main advantages of Caputo's fractional derivatives is that the initial conditions for fractional differential equations with such derivatives have a similar form as for integer-order differential equations, the validity of (7) can be verified using similar steps as in the integer-order models.

Define the norm $\|\phi\|_{\tau}=\sup _{s \in[-\tau, 0]}\|\phi(s)\|$ of the function $\phi \in P C B\left[[-\tau, 0], \mathbb{R}^{n}\right]$.

It is well known $[1,2,4,7-9,13,21,23]$ that Lyapunov functions can be a successful instrument in the investigation of the stability properties of a Lotka-Volterra type system. To apply the Lyapunov function method, we introduce the following notations:

$G_{k}=\left(t_{k-1}, t_{k}\right) \times \mathbb{R}_{+}^{n}, k=1,2, \ldots ; G=\cup_{k=1}^{\infty} G_{k} ;$

$V_{0}=\left\{V:[0, \infty) \times \mathbb{R}_{+}^{n} \rightarrow \mathbb{R}_{+}: V \in C\left[G, \mathbb{R}_{+}\right], t \in[0, \infty), V\right.$ is locally Lipschitzian in $y \in \mathbb{R}_{+}^{n}$ on each of the sets $G_{k}, V\left(t_{k}^{-}, y\right)=V\left(t_{k}, y\right)$ and $V\left(t_{k}^{+}, y\right)=\lim _{\substack{t \rightarrow t_{k} \\ t>t_{k}}} V(t, y)$ exists $\}$.

For $V \in V_{0}$ and $t \in\left[t_{k-1}, t_{k}\right)$, we will use the following fractional derivative of $V$ of order $q$, $0<q<1$ with respect to system (1) defined [39] by

$$
{ }^{C} D_{+}^{q} V(t, \phi(0))=\lim _{h \rightarrow 0^{+}} \sup \frac{1}{h^{q}}\left[V(t, \phi(0))-V\left(t-h, \phi(0)-h^{q} f(t, \phi)\right)\right],
$$

where $f(t, \phi)=\left(f_{1}(t, \phi), f_{2}(t, \phi), \ldots, f_{n}(t, \phi)\right)^{T}$ denotes the right-hand side of (5), $\phi \in P C B\left[[-\tau, 0], \mathbb{R}^{n}\right]$,

$$
f_{i}(t, \phi)=\phi_{i}(t) r_{i}(t)\left[1-\frac{\phi_{i}\left(t-\tau_{i i}\right)}{a_{i}(t)+\sum_{\substack{j=1 \\ j \neq i}}^{n} b_{j}(t) \phi_{j}\left(t-\tau_{i j}\right)}-c_{i}(t) \phi_{i}\left(t-\tau_{i j}\right)\right] .
$$

We also recall the following basic comparison result of fractional calculus from Reference [39].

Lemma 1. Assume that the function $V \in V_{0}$ is such that for $t \in \mathbb{R}_{+}, \phi \in \mathcal{P C}$,

$$
V\left(t^{+}, \phi(0)+\Delta \phi\right) \leq V(t, \phi(0)), \quad t=t_{k}
$$

and for a constant $\mu>0$ the inequality

$$
{ }^{C} D_{+}^{q} V(t, \phi(0)) \leq-\mu V(t, \phi(0)), t \neq t_{k}, k=1,2, \ldots
$$

is valid whenever $V(t+s, \phi(s)) \leq V(t, \phi(0))$ for $-\tau \leq s \leq 0$.

Then $\sup _{-\tau \leq s \leq 0} V\left(s, \phi_{0}(s)\right) \leq V(t, \phi(0))$ implies

$$
V\left(t, y\left(t ; 0, \phi_{0}\right)\right) \leq \sup _{-\tau \leq s \leq 0} V\left(0^{+}, \phi_{0}(s)\right) E_{q}\left(-\mu t^{q}\right), t \in[0, \infty),
$$

where $E_{q}$ is the Mittag-Leffler function defined as $E_{q}(z)=\sum_{\kappa=0}^{\infty} \frac{z^{\kappa}}{\Gamma(q \kappa+1)}, q>0$.

For more comparison results and properties on Mittag-Leffler functions we refer to References [35-37,39].

\section{Mittag-Leffler Stability Results}

Let $\phi_{0}^{*} \in P C B\left[[-\tau, 0], \mathbb{R}_{+}^{n}\right], \phi_{0}^{*}=\left(\phi_{10}^{*}, \phi_{20}^{*}, \ldots, \phi_{n 0}^{*}\right)^{T}$ and $y^{*}(t)=y^{*}\left(t ; 0, \phi_{0}^{*}\right)=\left(y_{1}^{*}\left(t ; 0, \phi_{0}^{*}\right)\right.$, $\left.y_{2}^{*}\left(t ; 0, \phi_{0}^{*}\right), \ldots, y_{n}^{*}\left(t ; 0, \phi_{0}^{*}\right)\right)^{T}$ be the solution of the impulsive control system (5) with initial function $\phi_{0}^{*}$. In the next, we shall suppose that $\phi_{i 0}^{*}(s) \geq 0, \sup \phi_{i 0}^{*}(s)<\infty, \phi_{i 0}^{*}(0)>0, \quad i=1,2, \ldots, n$. 
We will use the following definition for Mittag-Leffler stability of the state $y^{*}(t)$ of the model (5) [45-54].

Definition 1. The state $y^{*}(t)$ of system (5) is said to be globally Mittag-Leffler stable, if

$$
\left\|y\left(t ; 0, \phi_{0}\right)-y^{*}\left(t ; 0, \phi_{0}^{*}\right)\right\| \leq\left\{m\left[\left\|\phi_{0}-\phi_{0}^{*}\right\|_{\tau}\right] E_{q}\left(-\mu t^{q}\right)\right\}^{d},
$$

where $E_{q}$ is the corresponding Mittag-Leffler function, $q \in(0,1), \mu>0, d>0, m(0)=0, m(\phi) \geq 0$, and $m(\phi)$ is locally Lipschitz with respect to $\phi \in P C B\left[[-\tau, 0], \mathbb{R}^{n}\right]$.

In some of the literature [50], the constant $\mu$ is also called the degree of Mittag-Leffler state estimator, which can be considered as an equivalence of the convergence rate as state estimator error tends to zero when time $t$ goes to infinity.

Our main result in this section is the next theorem.

Theorem 1. Assume that:

1. The model's parameters satisfy

$$
2 m\left(\min _{1 \leq i \leq n} \frac{r_{i}^{L}}{a_{i}^{M}+M \sum_{\substack{j=1 \\ j \neq i}}^{n} b_{j}^{M}}+\min _{1 \leq i \leq n} r_{i}^{L} c_{i}^{L}\right)>\max _{1 \leq i \leq n} r_{i}^{M}+\max _{1 \leq i \leq n} \sum_{\substack{j=1 \\ j \neq i}}^{n} \frac{b_{j}^{M} r_{i}^{M} M^{2}}{\left(a_{i}^{L}+m \sum_{\substack{k=1 \\ \kappa \neq j}}^{n} b_{\kappa}^{L}\right)^{2}} .
$$

2. The impulsive functions $I_{i k}$ are such that

$$
I_{i k}\left(y_{i}\left(t_{k}\right)\right)=-\gamma_{i k}\left(y_{i}\left(t_{k}\right)-y_{i k}^{*}\left(t_{k}\right)\right), 0<\gamma_{i k}<2, i=1,2, \ldots n, k=1,2, \ldots
$$

Then, the state $y^{*}(t)$ of system (5) is globally Mittag-Leffler stable.

Proof. The proof is based on the Lyapunov-Razumikhin technique [39]. We consider the following Lyapunov function candidate

$$
V(t, y)=\sum_{i=1}^{n}\left|y_{i}-y_{i}^{*}\right|
$$

For $k=1, \ldots$, and $\phi \in P C B$, according to (8), we have

$$
\begin{aligned}
& V\left(t_{k}^{+}, \phi(0)+I_{k}(\phi)\right)=\sum_{i=1}^{n}\left|\phi_{i 0}(0)+I_{i k}\left(\phi_{0}\right)-\phi_{i 0}^{*}(0)-I_{i k}\left(\phi_{0}^{*}\right)\right| \\
& \leq \sum_{i=1}^{n}\left|\left(1-\gamma_{i k}\right)\left(\phi_{i 0}(0)-\phi_{i 0}^{*}(0)\right)\right| \leq V\left(t_{k}, \phi(0)\right) .
\end{aligned}
$$


Also, for $t \geq 0, t \neq t_{k}, k=1,2, \ldots$, and $\phi \in P C B$, we estimate the derivative ${ }^{C} D_{+}^{q} V(t, \phi(0))$ along the system (5), and get that

$$
\begin{aligned}
& { }^{C} D_{+}^{q} V(t, \phi(0)) \leq \max _{1 \leq i \leq n} r_{i}^{M} \sum_{i=1}^{n}\left|\phi_{i 0}(0)-\phi_{i 0}^{*}(0)\right|-\min _{1 \leq i \leq n} 2 r_{i}^{L} c_{i}^{L} m \sum_{i=1}^{n}\left|\phi_{i 0}(0)-\phi_{i 0}^{*}(0)\right| \\
& -\min _{1 \leq i \leq n} \frac{2 r_{i}^{L} m}{a_{i}^{M}+M \sum_{j=1}^{n} b_{j}^{M}} \sum_{i=1}^{n}\left|\phi_{i 0}(0)-\phi_{i 0}^{*}(0)\right| \\
& +\max _{1 \leq i \leq n} \sum_{\substack{j=1 \\
j \neq i}}^{n} \frac{b_{j}^{M} r_{i}^{M} M_{i}^{M}}{\left(a_{i}^{L}+m \sum_{\substack{\kappa=1 \\
\kappa \neq j}}^{n} b_{\kappa}^{L}\right)^{2}} \sum_{i=1}^{n} \sup _{s \in[-\tau, 0]}\left|\phi_{i 0}(s)-\phi_{i 0}^{*}(s)\right| \\
& \leq\left(-2 m\left(\min _{1 \leq i \leq n} \frac{r_{i}^{L}}{a_{i}^{M}+M \sum_{\substack{j=1 \\
j \neq i}}^{n} b_{j}^{M}}+\min _{1 \leq i \leq n} r_{i}^{L} c_{i}^{L}\right)\right. \\
& \left.+\max _{1 \leq i \leq n} r_{i}^{M}+\max _{1 \leq i \leq n} \sum_{\substack{j=1 \\
j \neq i}}^{n} \frac{b_{j}^{M \neq i} r_{i}^{M} M^{2}}{\left(a_{i}^{L}+m \sum_{\substack{\kappa=1 \\
\kappa \neq j}}^{n} b_{\kappa}^{L}\right)^{2}}\right) V(t, \varphi(0))
\end{aligned}
$$

is valid whenever $V(t+s, \varphi(s)) \leq V(t, \varphi(0))$ for $-\tau \leq s \leq 0$.

In view of (10) and condition 1 of Theorem 1 , there exists a positive $\mu$ such that

$$
{ }^{C} D_{+}^{q} V(t, \phi(0)) \leq-\mu V(t, \phi(0)), t \neq t_{k}, k=1,2, \ldots,
$$

whenever $V(t+s, \phi(s)) \leq V(t, \phi(0))$ for $-\tau \leq s \leq 0$.

From (9) and (11), by Lemma 1, it follows that

$$
V\left(t, y\left(t ; 0, \phi_{0}\right)\right) \leq \sup _{-\tau \leq s \leq 0} V\left(0^{+}, \phi_{0}(s)\right) E_{q}\left(-\mu t^{q}\right), t \in[0, \infty) .
$$

So,

$$
\left\|y(t)-y^{*}(t)\right\| \leq\left\|\phi_{0}-\phi_{0}^{*}\right\|_{\tau} E_{q}\left(-\mu t^{q}\right), t \in[0, \infty),
$$

and this completes the proof of the theorem.

Remark 1. The assumptions of Theorem 1 guarantee the Mittag-Leffler stability of the states of the impulsive control system (5). The impulsive control strategy is designed by (8). Therefore, the proposed structure of the control law can be applied to design impulsive based observers to estimate the states of (5) and globally Mittag-Leffler synchronize the states of the system (5) onto that of system (2). Note that, the synchronization scheme is independent on the lengths of the impulsive intervals.

Remark 2. One of the states that is of a great interest to researchers of Lotka-Volterra and related models are the so called "steady" or equilibrium states [1,3,21,47]. Let

$$
y^{*}=\left(y_{1}^{*}, y_{2}^{*}, \ldots, y_{n}^{*}\right)^{T}
$$

be the equilibrium of the control system (5) such that

$$
1=\frac{y_{i}^{*}}{a_{i}(t)+\sum_{\substack{j=1 \\ j \neq i}}^{n} b_{j}(t) y_{j}^{*}}-c_{i}(t) y_{i}^{*}, t \neq t_{k}
$$




$$
I_{i k}\left(y_{i}^{*}\right)=0, \quad i=1,2, \ldots, n, k=1,2, \ldots
$$

Since $I_{i k}\left(y_{i}^{*}\right)=0, \quad i=1,2, \ldots, n, k=1,2, \ldots$, the state $y^{*}$ is also a steady state for the controlled model (2). Hence, in the case when $y^{*}(t)=y^{*}=$ const, the providing results in Theorem 1 can be used so by means of convenient impulsive control strategy to keep the Mittag-Leffler stability properties of the equilibrium states of system (2). Note that the Mittag-Leffler stability for fractional-order models corresponds to the exponential stability for integer-order systems, which guarantees the fast convergence rate.

\section{Practical Stability Results}

In this section, following Reference [56] we will provide practical stability results for the impulsive control model (5).

We introduce the following definition.

Definition 2. The state $y^{*}(t)$ of the fractional impulsive control system (5) is said to be:

(a) practically uniformly globally stable with respect to $(\lambda, A)$, if given $(\lambda, A)$ with $0<\lambda<A$, we have $\left\|\phi_{0}-\phi_{0}^{*}\right\|_{\tau}<\lambda$ implies

$$
\left\|y\left(t ; 0, \phi_{0}\right)-y^{*}\left(t ; 0, \phi_{0}^{*}\right)\right\| \leq A, t \geq 0 ;
$$

(b) practically uniformly globally Mittag-Leffler stable with respect to $(\lambda, A)$, if given $(\lambda, A)$ with $0<\lambda<A$, we have $\left\|\phi_{0}-\phi_{0}^{*}\right\|_{\tau}<\lambda$ implies

$$
\left\|y\left(t ; 0, \phi_{0}\right)-y^{*}\left(t ; 0, \phi_{0}^{*}\right)\right\| \leq A+v\left\|\phi_{0}-\phi_{0}^{*}\right\|_{\tau} E_{q}\left[-\mu t^{q}\right], t \geq 0, v, \mu>0 .
$$

Condition 1 of Theorem 1 guarantees the existence of a positive constant $\mu$ such that (11) holds. The application of (11) together with the appropriate choice of the Lyapunov-candidate function $V$ imply the global Mittag-Leffler stability of the state $y^{*}(t)$ of the control system (5) by means of the designed impulsive control law (8). This section will firstly address the case, when $\mu=0$ and the state $y^{*}(t)$ is not globally Mittag-Leffler stable. We will prove that in such case the impulsive controller (8) may assure its practical uniform global stability.

We will use again the Lyapunov function candidate

$$
V(t, y)=\sum_{i=1}^{n}\left|y_{i}-y_{i}^{*}\right|
$$

and functions of the following class:

$K=\left\{a \in C\left[\mathbb{R}_{+}, \mathbb{R}_{+}\right]: a(u)\right.$ is strictly increasing in $u$ and $\left.a(0)=0\right\}$.

Theorem 2. Assume that the impulsive controller satisfies (8) and:

1. $0<\lambda<A$ are given.

2. The model's parameters satisfy

$$
2 m\left(\min _{1 \leq i \leq n} \frac{r_{i}^{L}}{a_{i}^{M}+M \sum_{\substack{j=1 \\ j \neq i}}^{n} b_{j}^{M}}+\min _{1 \leq i \leq n} r_{i}^{L} c_{i}^{L}\right)=\max _{1 \leq i \leq n} r_{i}^{M}+\max _{1 \leq i \leq n} \sum_{\substack{j=1 \\ j \neq i}}^{n} \frac{b_{j}^{M} r_{i}^{M} M^{2}}{\left(a_{i}^{L}+m \sum_{\substack{\kappa=1 \\ \kappa \neq j}}^{n} b_{\kappa}^{L}\right)^{2}} .
$$

3. There exists a function $b \in K$ such that

$$
V(t, y) \leq b\left(\left\|y-y^{*}\right\|\right), t \in \mathbb{R}_{+}, y, y^{*} \in \mathbb{R}^{n} .
$$

4. $b(\lambda)<$ A holds.

Then, the state $y^{*}(t)$ of system (5) is practically uniformly globally stable with respect to $(\lambda, A)$. 
Proof. Let $0<\lambda<A$. We will prove that $\left\|\phi_{0}-\phi_{0}^{*}\right\|_{\tau}<\lambda$ implies $\left\|y\left(t ; 0, \phi_{0}\right)-y^{*}\left(t ; 0, \phi_{0}^{*}\right)\right\| \leq A, t \geq 0$.

If this is not true, there exists a corresponding solution $y\left(t ; 0, \phi_{0}\right)$ of (5) with $\left\|\phi_{0}-\phi_{0}^{*}\right\|_{\tau}<\lambda$, and $0<t^{*}<\infty, t_{k}<t^{*} \leq t_{k+1}$ for some $k$, such that

$$
\left\|y\left(t ; 0, \phi_{0}\right)-y^{*}\left(t ; 0, \phi_{0}^{*}\right)\right\|<A, t \in\left[0, t_{k}\right] \text { and }\left\|y\left(t^{*}\right)-y^{*}\left(t^{*}\right)\right\| \geq A .
$$

Note that the impulsive control low (8) guarantees that if

$$
\left\|y\left(t_{k} ; 0, \phi_{0}\right)-y^{*}\left(t_{k} ; 0, \phi_{0}^{*}\right)\right\|<A,
$$

then

$$
\begin{gathered}
|| y\left(t_{k}^{+} ; 0, \phi_{0}\right)-y^{*}\left(t_{k}^{+} ; 0, \phi_{0}^{*}\right)||=\sum_{i=1}^{n}\left|y_{i}\left(t_{k}\right)+I_{i k}\left(y_{i}\left(t_{k}\right)\right)-y_{i}^{*}\left(t_{k}\right)-I_{i k}\left(y_{i}^{*}\left(t_{k}\right)\right)\right| \\
\leq \sum_{i=1}^{n}\left|\left(1-\gamma_{i k}\right)\left(y_{i}\left(t_{k}\right)-y_{i}^{*}\left(t_{k}\right)\right)\right|<A .
\end{gathered}
$$

Hence $t^{*} \neq t_{k}$ for any $k=1,2, \ldots$.

From condition 2 of Theorem 2 by similar arguments as in the proof of Theorem 1, we have

$$
{ }^{C} D_{+}^{q} V(t, \phi(0)) \leq 0, t \neq t_{k}, k=1,2, \ldots,
$$

whenever $V(t+s, \phi(s)) \leq V(t, \phi(0))$ for $-\tau \leq s \leq 0$.

Also, using (12) and (8), we obtain by Lemma 1, for $\mu=0$

$$
V\left(t, y\left(t ; 0, \phi_{0}\right)\right) \leq \sup _{-\tau \leq s \leq 0} V\left(0^{+}, \phi_{0}(s)\right), t \in\left[0, t^{*}\right] .
$$

Hence, (13), (8) and condition 4 of Theorem 2 imply

$$
A \leq V\left(t^{*}, y\left(t^{*} ; 0, \phi_{0}\right)\right) \leq \sup _{-\tau \leq s \leq 0} V\left(0^{+}, \phi_{0}(s)\right) \leq b(\lambda)<A .
$$

The contradiction obtained proves our claim that system (5) is practically uniformly globally stable with respect to $(\lambda, A)$.

Remark 3. In Theorem 2 we proved that even in some concrete cases, when the system (5) is not globally Mittag-Leffler stable, by the impulsive controller (8) we can made the vicinity of a state $y^{*}$ arbitrarily small so that all other states to remain arbitrarily close to it provided that their initial functions were sufficiently near the initial data of the particular state $y^{*}$. The property of practical stability is very important in many applications when the Mittag-Leffler stability is conservative [58,60,61]. For type (2) models from the population biology this can compensate a not stable initial population by keeping the population densities between particular bounds for $t \geq 0$.

Theorem 3. Assume that:

1. There exists a function $V \in V_{0}$ for which conditions of Lemma 1 are met.

2. The function $V(t, y)$ is such that

$$
\left\|y-y^{*}\right\|-A \leq V(t, y) \leq a(u)\left\|y-y^{*}\right\|, t \in \mathbb{R}_{+}, y, y^{*} \in \mathbb{R}^{n},
$$

where the continuous function $a(u) \geq 1$ exists for any $u>0$.

3. Condition 1 of Theorem 2 holds.

Then, the state $y^{*}(t)$ of system (5) is practically uniformly globally Mittag-Leffler stable with respect to $(\lambda, A)$. 
Proof. Let $0<\lambda<A$, and $\left\|\phi_{0}-\phi_{0}^{*}\right\|_{\tau}<\lambda$ for an initial function $\phi_{0} \in P C B\left[[-\tau, 0], \mathbb{R}^{n}\right]$ of an arbitrary solution $y(t)=y\left(t ; 0, \phi_{0}\right)$ of the initial value problem (5), (6).

From Lemma 1, we have that

$$
V\left(t, y\left(t ; 0, \phi_{0}\right)\right) \leq \sup _{-\tau \leq s \leq 0} V\left(0^{+}, \phi_{0}(s)\right) E_{q}\left(-\mu t^{q}\right), t \in[0, \infty),
$$

where $\mu>0$.

From (14) and condition 2 of Theorem 3, we have

$$
\begin{gathered}
\left\|y\left(t ; 0, \phi_{0}\right)-y^{*}\left(t ; 0, \phi_{0}^{*}\right)\right\|-A \leq V\left(t, y\left(t ; 0, \phi_{0}\right)\right) \\
\leq \sup _{-\tau \leq s \leq 0} V\left(0^{+}, \phi_{0}(s)\right) E_{q}\left(-\mu t^{q}\right) \leq a(u)\left\|\phi_{0}-\phi_{0}^{*}\right\|_{\tau} E_{q}\left(-\mu t^{q}\right), \quad t \geq 0 .
\end{gathered}
$$

Therefore, for any $v \geq a(u), u>0$, we have

$$
\left\|y\left(t ; 0, \phi_{0}\right)-y^{*}\left(t ; 0, \phi_{0}^{*}\right)\right\| \leq A+v\left\|\phi_{0}-\phi_{0}^{*}\right\|_{\tau} E_{q}\left(-\mu t^{q}\right), \quad t \geq 0,
$$

which shows that system (5) is practically uniformly globally Mittag-Leffler stable with respect to $(\lambda, A)$.

Remark 4. For $A=0$ Theorem 3 implies global Mittag-Leffler stability. However, Theorem 3 can be applied when this stability notion is pointless from the applied point of view. In such cases the designed impulsive controller allows the practical uniform global Mittag-Leffler stability.

Remark 5. More detailed explanations about the importance of the practical stability notion for applied problems, as well as, for relations between stability and practical stability concepts can be found in References [56,61,64].

\section{Stability of Sets}

In this Section, we extend the Lyapunov function method to stability of sets criteria for the impulsive control system (5). In fact, considering closed sets of states as attractors instead of equilibrium states is common in population dynamics [1].

Let $B \subset \mathbb{R}^{n}$ be an arbitrary set (compact or not compact). Define the norms:

$\|y\|^{B}=\inf \{\|y-z\|: z \in B\}$ is the distance from $y \in \mathbb{R}^{n}$ to $B$;

$\|\phi\|_{\tau}^{B}=\sup _{s \in[-\tau, 0]}\|\phi(s)\|^{B}$ is the distance between a function $\phi \in P C B\left[[-\tau, 0], \mathbb{R}^{n}\right]$ and $B$.

Definition 3. The solutions of system (5) are said to be uniformly bounded with respect to the set $B$, if for any positive constant $\eta>0$ there exists a constant $\beta>0$ such that $\phi_{0} \in P C B\left[[-\tau, 0], \mathbb{R}^{n}\right]$ and $\left\|\phi_{0}\right\|_{\tau}^{B} \leq \eta$ imply

$$
\left\|y\left(t ; 0, \phi_{0}\right)\right\|^{B} \leq \beta, t \geq 0 .
$$

Definition 4. The set B is said to be globally Mittag-Leffler stable with respect to system (5), if the solutions of system (5) are uniformly bounded with respect to the set $B$, and

$$
\left\|y\left(t ; 0, \phi_{0}\right)\right\|^{B} \leq\left\{m\left[\left\|\phi_{0}\right\|_{\tau}^{B}\right] E_{q}\left(-\mu t^{q}\right)\right\}^{d},
$$

where $E_{q}$ is the corresponding Mittag-Leffler function, $q \in(0,1), \mu>0, d>0, m(0)=0, m(\phi) \geq 0$, and $m(\phi)$ is locally Lipschitz with respect to $\phi \in P C B\left[[-\tau, 0], \mathbb{R}^{n}\right]$.

Remark 6. The notion of stability of sets with respect to system (5) generalizes the stability of single states concepts of system (5). For example, when $B=\left\{y \in \mathbb{R}^{n}: y=y^{*}(t), t \geq 0\right\}$, Definition 3 reduces to Definition 1 for the global Mittag-Leffler stability of the state $y^{*}(t), t \geq 0$. 
Remark 7. The requirement for uniform boundedness of all solutions of (5) with respect to $B$ is required by the fact that for noncompact sets $B$ there is an opportunity that some solutions may tend to infinity in finite time within the set. If the set $B$ is compact (for example, the equilibrium state), then the boundedness condition is surplus.

Theorem 4. Assume that:

1. There exists a function $V \in V_{0}$ such that $V(t, y)=0$ for $y \in B$ and $V(t, y)>0$ for $y \in \mathbb{R}_{+}^{n} \backslash B, t \geq 0$.

2. There exists a function $b \in K$ such that

$$
\|y\|^{B} \leq V(t, y) \leq b\left(\|y\|^{B}\right),(t, y) \in \mathbb{R}_{+} \times \mathbb{R}_{+}^{n} .
$$

3. For the function $V$ conditions of Lemma 1 are met.

Then, the set B is globally Mittag-Leffler stable with respect to system (5).

Proof. We will first prove the uniform boundedness of the solutions of (5) with respect to the set $B$. Let $\eta>0$ be given and let $\phi_{0} \in P C B\left[[-\tau, 0], \mathbb{R}^{n}\right]$ be such that $\left\|\phi_{0}\right\|_{\tau}^{B} \leq \eta$. We can choose $\beta>0$ so that $b(\eta)<\beta$.

Let $y\left(t ; 0, \phi_{0}\right)$ be the solution of the system (5) corresponding to the initial function $\phi_{0}$.

Since the conditions of Lemma 1 are met, then for $\mu=0$, we have

$$
V\left(t, y\left(t ; 0, \phi_{0}\right)\right) \leq \sup _{-\tau \leq s \leq 0} V\left(0^{+}, \phi_{0}(s)\right), t \geq 0 .
$$

Now, condition 2 of Theorem 4 and (15) imply the inequalities

$$
\begin{gathered}
\left\|y\left(t ; 0, \phi_{0}\right)\right\|^{B} \leq V\left(t, y\left(t ; 0, \phi_{0}\right)\right) \leq \sup _{-\tau \leq s \leq 0} V\left(0^{+}, \phi_{0}(s)\right) \\
\leq b\left(\left\|\phi_{0}\right\|_{\tau}^{B}\right) \leq b(\eta)<\beta, t \geq 0 .
\end{gathered}
$$

Therefore,

$$
\left\|y\left(t ; 0, \phi_{0}\right)\right\|^{B} \leq \beta, t \geq 0 .
$$

Since the solution $y\left(t ; 0, \phi_{0}\right)$ is arbitrary the above proves the uniform boundedness of the solutions of (5) with respect to the set $B$.

In a similar way, as above, using Lemma 1 and condition 2 of Theorem 4, we obtain

$$
\begin{gathered}
\left\|y\left(t ; 0, \phi_{0}\right)\right\|^{B} \leq V\left(t, y\left(t ; 0, \phi_{0}\right)\right) \leq \sup _{-\tau \leq s \leq 0} V\left(0^{+}, \phi_{0}(s)\right) E_{q}\left(\mu t^{q}\right) \\
\leq b\left(\left\|\phi_{0}\right\|_{\tau}^{B}\right) E_{q}\left(\mu t^{q}\right), t \geq 0,
\end{gathered}
$$

which according to Definition 4 means that the set B is globally Mittag-Leffler stable set with respect to (5).

Remark 8. Theorem 4 shows that the impulsive control law (8) is designed so that gets global Mittag-Leffler stability of a set B with respect to the fractional model (5). For the integer-order systems this is equivalent to the fact that all trajectories arrive at a given target set $B$. Hence, the proposed result is of a great importance in the cases when the information on the system's parameters is incomplete and ones has to conduct a robust stability analysis.

Remark 9. It is well know that if B is globally asymptotically stable with respect to (5), then the trivial solution of (5) is practically stable [65]. 
Remark 10. We can also define the hybrid concept of practical stability of an arbitrary set B with respect to the impulsive control system (5). In fact, this concept is more natural than that of a neighbourhood of a state. In this case, one important question will be how well the stability behavior can be impulsively controlled, which is a subject for our future research.

\section{Examples and Simulations}

Example 1. Consider the following two-species fractional cooperation Lotka-Volterra system

$$
\left\{\begin{array}{l}
{ }^{C} D_{t}^{q} x_{1}(t)=x_{1}(t)\left[1-\frac{x_{1}\left(t-\tau_{11}\right)}{1+5 x_{2}\left(t-\tau_{12}\right)}-3 x_{1}\left(t-\tau_{12}\right)\right], \\
{ }^{C} D_{t}^{q} x_{2}(t)=x_{2}(t)\left[1-\frac{x_{2}\left(t-\tau_{22}\right)}{0.3+0.7 x_{1}\left(t-\tau_{21}\right)}-3 x_{2}\left(t-\tau_{21}\right)\right],
\end{array}\right.
$$

$t \geq 0,0 \leq \tau_{i j} \leq 1, i, j=1,2$.

We can easily check that the point $\left(\frac{2}{7}, \frac{1}{5}\right)$ is an equilibrium for the model (16).

The corresponding fractional control Lotka-Volterra system is given by

$$
\left\{\begin{array}{l}
{ }^{C} D_{t}^{q} y_{1}(t)=y_{1}(t)\left[1-\frac{y_{1}\left(t-\tau_{11}\right)}{1+5 y_{2}\left(t-\tau_{12}\right)}-3 y_{1}\left(t-\tau_{12}\right)\right], t \neq t_{k} \\
{ }^{C} D_{t}^{q} y_{2}(t)=y_{2}(t)\left[1-\frac{y_{2}\left(t-\tau_{22}\right)}{0.3+0.7 y_{1}\left(t-\tau_{21}\right)}-3 y_{2}\left(t-\tau_{21}\right)\right], t \neq t_{k}
\end{array}\right.
$$

under impulsive control law

$$
\left\{\begin{aligned}
\Delta y_{1}\left(t_{k}\right) & =y_{1}\left(t_{k}^{+}\right)-y_{1}\left(t_{k}\right)=-\frac{2}{5}\left(y_{1}\left(t_{k}\right)-\frac{2}{7}\right), k=1,2, \ldots \\
\Delta y_{2}\left(t_{k}\right) & =y_{2}\left(t_{k}^{+}\right)-y_{2}\left(t_{k}\right)=-\frac{1}{3}\left(y_{2}\left(t_{k}\right)-\frac{1}{5}\right), k=1,2, \ldots
\end{aligned}\right.
$$

where $0<t_{1}<t_{2}<\ldots$ and $\lim _{k \rightarrow \infty} t_{k}=\infty$.

We have that for $y_{1}^{*}=\frac{2}{7}$ and $y_{2}^{*}=\frac{1}{5},{ }^{C} D_{t}^{q} y_{1}^{*}=0$ and ${ }^{C} D_{t}^{q} y_{2}^{*}=0$, that is, the state $\left(\frac{2}{7}, \frac{1}{5}\right)$ is an equilibrium for the model (17), (18) too.

Also, obviously all conditions of Theorem 1 are satisfied for $m=\frac{1}{5}, M=\frac{2}{7}, a_{1}^{L}=a_{1}^{M}=1, a_{2}^{L}=a_{2}^{M}=0.3$, $b_{1}^{L}=b_{1}^{M}=0.7, b_{2}^{L}=b_{2}^{M}=5, c_{i}^{L}=c_{i}^{M}=3, i=1,2, \gamma_{1 k}=\frac{2}{5}, \gamma_{2 k}=\frac{1}{3}, k=1,2, \ldots$. In fact, we have that

$$
1.364 \approx 2 m\left(\min _{1 \leq i \leq 2} \frac{r_{i}^{L}}{a_{i}^{M}+M \sum_{\substack{j=1 \\ j \neq i}}^{n} b_{j}^{M}}+\min _{1 \leq i \leq 2} r_{i}^{L} c_{i}^{L}\right)>\max _{1 \leq i \leq 2} r_{i}^{M}+\max _{1 \leq i \leq 2} \sum_{\substack{j=1 \\ j \neq i}}^{2} \frac{b_{j}^{M} r_{i}^{M} M^{2}}{\left(a_{i}^{L}+m \sum_{\substack{\kappa=1 \\ \kappa \neq j}}^{2} b_{\kappa}^{L}\right)^{2}} \approx 1.314 .
$$

Hence, the equilibrium state $\left(\frac{2}{7}, \frac{1}{5}\right)$ of the impulsive system (17), (18) is globally Mittag-Leffler stable. This example shows the effectiveness of the obtained results under an impulsive controller (18) of type (8). It also, demonstrates that the proposed sufficient conditions are reasonably strong. The global Mittag-Leffler stable behavior of the state variables $y_{1}(t)$ and $y_{2}(t)$ is demonstrated on Figure 1 for $q=0.4, x_{1}(s)=y_{1}(s)=2$, $x_{2}(s)=y_{2}(s)=3, s \in[-0.01,0], y_{1}\left(0^{+}\right)=2, y_{2}\left(0^{+}\right)=3$ and $t_{k}=[0.03,0.07,0.11,0.15,0.19,0.23]$. 


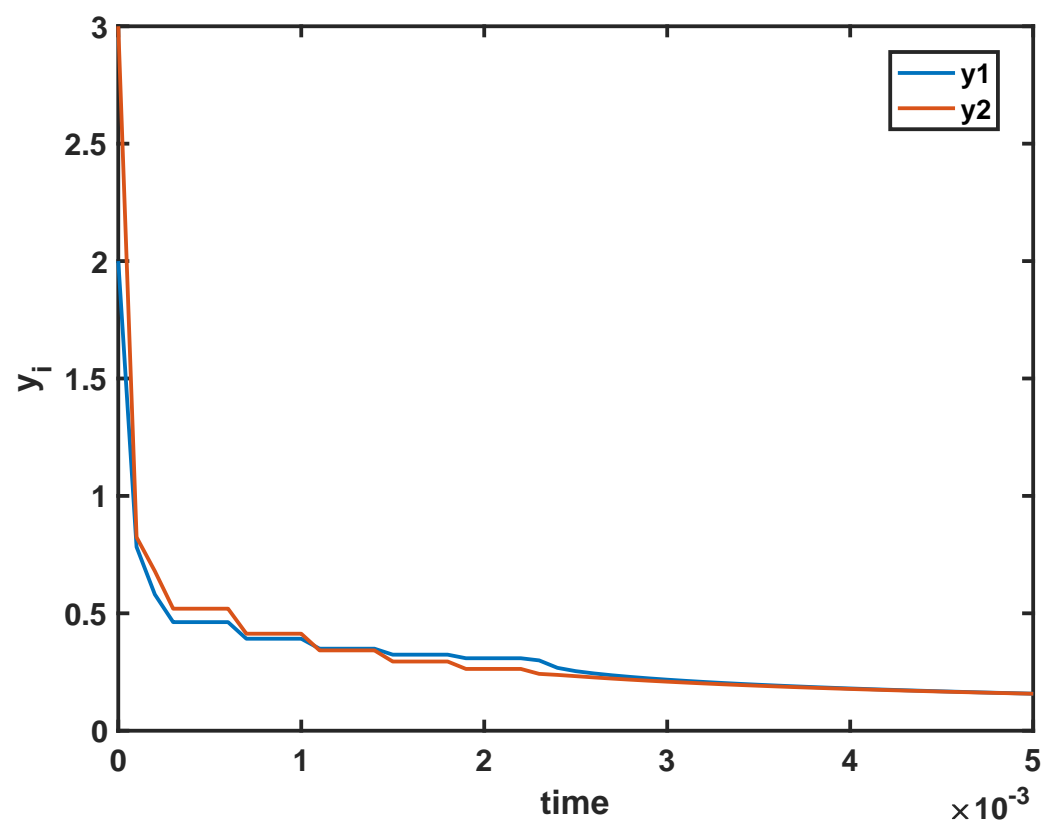

Figure 1. The global Mittag-Leffler stable behavior of the state variables $y_{1}(t)$ and $y_{2}(t)$ of the model (17) and (18).

Example 2. Consider again the fractional impulsive Lotka-Volterra system (17) and (18) with a globally Mittag-Leffler stable equilibrium state $y^{*}=\left(\frac{2}{7}, \frac{1}{5}\right)$. Let the region of the Mittag-Leffler stability be determined by

$$
\| \phi-\left.y^{*}\right|_{\tau}<k
$$

If $\lambda$ and $A$ are such that

$$
k<\lambda<A,
$$

then for an initial function $\phi_{0}=\left(\phi_{01}, \phi_{02}\right)^{T}$ such that

$$
k \leq\left\|\phi-y^{*}\right\|_{\tau}=\sup _{s \in[-\tau, 0]}\left\|\phi(s)-y^{*}\right\|=\sup _{s \in[-\tau, 0]}\left(\left|\phi_{01}(s)-\frac{2}{7}\right|+\left|\phi_{02}(s)-\frac{1}{5}\right|\right)<\lambda
$$

the state $\left(\frac{2}{7}, \frac{1}{5}\right)$ will not be practically uniformly globally Mittag-Leffler stable. Note that in the particular example, condition 2 of Theorem 2 is not satisfied.

The example again shows that Mittag-Leffler stability is not enough for practical Mittag-Leffler stability.

Example 3. Consider the two-species fractional cooperation Lotka-Volterra model

$$
\left\{\begin{array}{l}
{ }^{C} D_{t}^{q} x_{1}(t)=x_{1}(t)\left[1-\frac{x_{1}\left(t-\tau_{11}\right)}{1+5 x_{2}\left(t-\tau_{12}\right)}-\frac{13}{4} x_{1}\left(t-\tau_{12}\right)\right], \\
{ }^{C} D_{t}^{q} x_{2}(t)=x_{2}(t)\left[1-\frac{x_{2}\left(t-\tau_{22}\right)}{1+7 x_{1}\left(t-\tau_{21}\right)}-\frac{4}{3} x_{2}\left(t-\tau_{21}\right)\right],
\end{array}\right.
$$

$t \geq 0,0 \leq \tau_{i j} \leq 1, i, j=1,2$.

We can easily check that the point $\left(\frac{2}{7}, \frac{3}{5}\right)$ is an equilibrium for the model (19). However, since

$$
2 m\left(\min _{1 \leq i \leq 2} \frac{r_{i}^{L}}{a_{i}^{M}+M \sum_{\substack{j=1 \\ j \neq i}}^{n} b_{j}^{M}}+\min _{1 \leq i \leq 2} r_{i}^{L} c_{i}^{L}\right)<\max _{1 \leq i \leq 2} r_{i}^{M}+\max _{1 \leq i \leq 2} \sum_{\substack{j=1 \\ j \neq i}}^{2} \frac{b_{j}^{M} r_{i}^{M} M^{2}}{\left(a_{i}^{L}+m \sum_{\substack{\kappa=1 \\ \kappa \neq j}}^{2} b_{\mathcal{K}}^{L}\right)^{2}}
$$


we cannot make any conclusion about its global Mittag-Leffler stability.

For the corresponding fractional impulsive control Lotka-Volterra system

$$
\left\{\begin{array}{l}
{ }^{c} D_{t}^{q} y_{1}(t)=y_{1}(t)\left[1-\frac{y_{1}\left(t-\tau_{11}\right)}{1+5 y_{2}\left(t-\tau_{12}\right)}-\frac{13}{4} y_{1}\left(t-\tau_{12}\right)\right], t \neq t_{k} \\
{ }^{c} D_{t}^{q} y_{2}(t)=y_{2}(t)\left[1-\frac{y_{2}\left(t-\tau_{22}\right)}{1+7 y_{1}\left(t-\tau_{21}\right)}-\frac{4}{3} y_{2}\left(t-\tau_{21}\right)\right], t \neq t_{k} \\
\Delta y_{1}\left(t_{k}\right)=-\frac{2}{3}\left(y_{1}\left(t_{k}\right)-\frac{2}{7}\right), k=1,2, \ldots, \\
\Delta y_{2}\left(t_{k}\right)=-\frac{3}{7}\left(y_{2}\left(t_{k}\right)-\frac{3}{5}\right), k=1,2, \ldots
\end{array}\right.
$$

with $0<t_{1}<t_{2}<\ldots, \lim _{k \rightarrow \infty} t_{k}=\infty$ consider the Lyapunov function

$$
V(t, y)=\sum_{i=1}^{n}\left|y_{i}-y_{i}^{*}\right|
$$

Let $\lambda$ and $A$ be given, and $a(u)=u+1, u \in \mathbb{R}_{+}$. Then all conditions of Theorem 3 are satisfied, and hence, the equilibrium $\left(\frac{2}{7}, \frac{3}{5}\right)$ of $(20)$ is practically uniformly globally Mittag-Leffler stable, which means that the impulsive controllers contribute to keep the population densities of both spaces between given bounds. For example, in the case $q=0.4, t_{k}=[0.03,0.07,0.11,0.15,0.19,0.23], \tau_{i j}=0.01, i, j=1,2$, $x_{1}(s)=y_{1}(s)=2, x_{2}(s)=y_{2}(s)=3, s \in[-0.01,0], y_{1}\left(0^{+}\right)=2, y_{2}\left(0^{+}\right)=3$, the practical uniform global Mittag-Leffler stable behavior of the model (20) is shown in Figure 2.

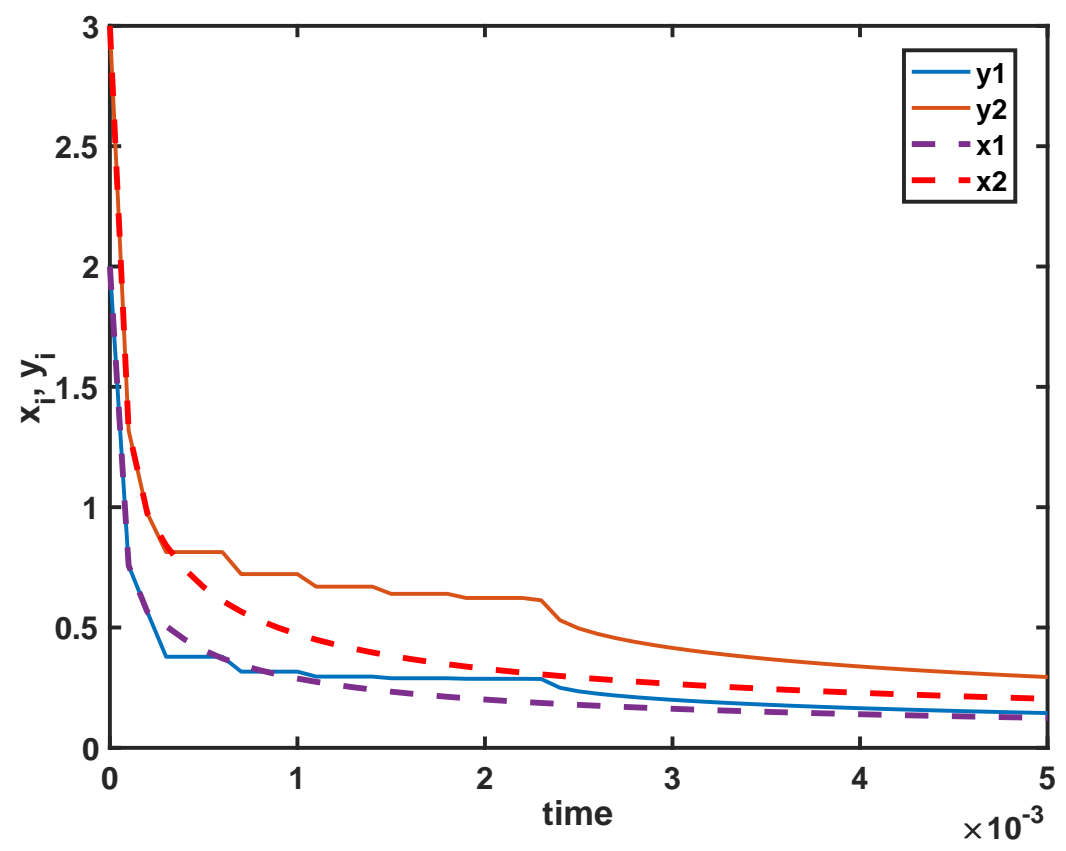

Figure 2. The practical uniform global Mittag-Leffler stable behavior of the model (20). The state variables $x_{1}(t)$ and $x_{2}(t)$ of (19) and $y_{1}(t)$ and $y_{2}(t)$ of (20).

Example 4. For the fractional impulsive Lotka-Volterra model (17), (18) consider the set

$$
B=\left\{z \in \mathbb{R}_{+}^{2}: 0 \leq z \leq y^{*}\right\},
$$

where $y^{*}=\left(y_{1}^{*}, y_{2}^{*}\right)=\left(\frac{2}{7}, \frac{1}{5}\right)$. 
Following the steps in the proof of Theorem 1 using the Lyapunov function

$$
V(t, y)=\|y\|^{B}
$$

it is trivial to show that the set B is globally Mittag-Leffler stable with respect to (17), (18).

\section{Conclusions}

In this paper, we introduce a fractional-order Lotka-Volterra type cooperation model. Based on Lyapunov function theory we present an impulsive control strategy that can be applied as a Mittag-Leffler stability and synchronization mechanism. In addition, more general practical stability results and stability of sets results with respect to the impulsive control system are established. Examples are presented to demonstrate the obtained criteria and the relations between the investigated stability behaviors. It is shown that the proposed impulsive control technique provides a useful tool for the design of appropriate population models. It can be also beneficial to models with reaction-diffusion terms which are the subject of our future research.

Author Contributions: Conceptualization, F.S. and I.S.; methodology, R.T., F.S. and I.S.; formal analysis, R.T., F.S. and I.S.; investigation, R.T., F.S. and I.S.; visualization, R.T.; writing-original draft preparation, I.S. All authors have read and agreed to the published version of the manuscript.

Funding: F.S. and R.T. partially funded by National Institutes of Health BRAIN Theories NIMH-NIBIB R01EB026939.

Conflicts of Interest: The authors declare no conflict of interest.

\section{References}

1. Ahmad, S.; Stamova, I.M. (Eds.) Lotka-Volterra and Related Systems: Recent Developments in Population Dynamics, 1st ed.; Walter de Gruyter: Berlin, Germany, 2013; ISBN 978-3-11-026951-2.

2. Gopalsamy, K. Stability and Oscillations in Delay Differential Equations of Population Dynamics, 1st ed.; Springer: Boston, MA, USA, 1992; ISBN 978-90-481-4119-7.

3. Takeuchi, Y. Global Dynamical Properties of Lotka-Volterra Systems, 1st ed.; World Scientific: Singapore, 1996; ISBN 9810224710/9789810224714.

4. Zhang, T.; Yang, L.; Xu, L. Stage-structured control on a class of predator-prey system in almost periodic environment. Intern. J. Control 2020, 93, 1442-1460. [CrossRef]

5. Wei, F.; Wang, K. Asymptotically periodic solution of $n$-species cooperation system with time delay. Nonlinear Anal. Real World Appl. 2006, 7, 591-596. [CrossRef]

6. May, R.M. Theoretical Ecology. Principles and Applications, 1st ed.; Saunders: Philadelphia, PA, USA, 1976; ISBN 0721662056/978-0721662053.

7. Chen, F.; Liao, X.; Huang, Z. The dynamic behavior of $N$-species cooperation system with continuous time delays and feedback controls. Appl. Math. Comput. 2006, 181, 803-815. [CrossRef]

8. $\mathrm{Xu}, \mathrm{R} . ;$ system with time delays. Indian J. Pure Appl. Math. 2004, 35, 915-936.

9. Yang, P.; Xu, R. Global asymptotic stability of periodic solution in $n$-species cooperative system with time delays. J. Biomath. 1998, 13, 841-846.

10. Baez, J.C.; Pollard, B.S. Relative entropy in biological systems. Entropy 2016, 18, 46. [CrossRef]

11. Rachdi, M.; Waku, D.; Hazgui, H.; Demongeot, J. Entropy as a robustness marker in genetic regulatory networks. Entropy 2020, 22, 260. [CrossRef]

12. finnegan A.; Song J.S. Maximum entropy methods for extracting the learned features of deep neural networks. PLoS Comput. Biol. 2017, 13, e1005836. [CrossRef]

13. Ahmad, S.; Stamova, I.M. Asymptotic stability of an $n$-dimensional impulsive competitive system. Nonlinear Anal. 2007, 8, 654-663. [CrossRef]

14. Ballinger, G.; Liu, X. Permanence of population growth models with impulsive effects. Math. Comput. Model. 1997, 26, 59-72. [CrossRef] 
15. Dai, C.; Zhao, M.; Chen, L. Complex dynamic behavior of three-species ecological model with impulse perturbations and seasonal disturbances. Math. Comput. Simulation 2012, 84, 83-97. [CrossRef]

16. Dong, L.; Chen, L.; Sun, L. Extinction and permanence of the predator-prey system with stocking of prey and harvesting of predator impulsively. Math. Methods Appl. Sci. 2006, 29, 415-425. [CrossRef]

17. Dong, L.; Takeuchi, Y. Impulsive control of multiple Lotka-Volterra systems. Nonlinear Anal. Real World Appl. 2013, 14, 1144-1154. [CrossRef]

18. Struk, O.O.; Tkachenko, V. On impulsive Lotka-Volterra systems with diffusion. Ukr. Math. J. 2002, 54, 629-646. [CrossRef]

19. Tang, S.; Chen, L. The periodic predator-prey Lotka-Volterra model with impulsive effects. J. Mech. Med. Biol. 2002, 2, 267-296. [CrossRef]

20. Yu, X.; Wang, Q.; Bai, Y. Permanence and almost periodic solutions for $N$-species nonautonomous Lotka-Volterra competitive systems with delays and impulsive perturbations on time scales. Complexity 2018, 2018, 2658745. [CrossRef]

21. Stamova, I.M. Impulsive control for stability of $n$-species Lotka-Volterra cooperation models with finite delays. Appl. Math. Lett. 2010, 23, 1003-1007. [CrossRef]

22. Haddad, W.M.; Chellaboina, V.S.; Nersesov, S.G. Impulsive and Hybrid Dynamical Systems, Stability, Dissipativity, and Control, 1st ed.; Princeton University Press: Princeton, NJ, USA, 2006; ISBN 9780691127156

23. Stamova, I.M.; Stamov, G.T. Applied Impulsive Mathematical Models, 1st ed.; Springer: Cham, Switzerland, 2016; ISBN 978-3-319-28060-8/978-3-319-28061-5.

24. Yang, X.; Peng, D.; Lv, X.; Li, X. Recent progress in impulsive control systems. Math. Comput. Simul. 2019, 155, 244-268. [CrossRef]

25. Abbas, S.; Banerjee, M.; Momani, S. Dynamical analysis of fractional-order modified logistic model. Comput. Math. Appl. 2011, 62, 1098-1104. [CrossRef]

26. Mohyud-Din, S.T.; Ali, A.; Bin-Mohsin, B. On biological population model of fractional order. Int. J. Biomath. 2016, 9, 1650070. [CrossRef]

27. Teka, W.; Marinov, T.; Santamaria, F. Neuronal spike timing adaptation described with a fractional leaky integrate-and-fire model. PLoS Comput. Biol. 2014, 10, e1003526. [CrossRef] [PubMed]

28. Vázquez-Guerrero, P.; Gómez-Aguilar, J.F.; Santamaria, F.; Escobar-Jiméneza, R.F. Design of a high-gain observer for the synchronization of chimera states in neurons coupled with fractional dynamics. Physical $A$ 2020, 539, 122896. [CrossRef]

29. Agrawal, S.K.; Srivastava, M.; Das, S, Synchronization between fractional-order Ravinovich-Fabrikant and Lotka-Volterra systems. Nonlinear Dyn. 2012, 69, 2277-2288. [CrossRef]

30. Das, S.K.; Gupta, P.K. A mathematical model on fractional Lotka-Volterra equations. J. Theor. Biol. 2011, 277, 1-6. [CrossRef]

31. Gatabazi, P.; Mba, J. C.; Pindza, E. Fractional gray Lotka-Volterra models with application to cryptocurrencies adoption. Chaos 2019, 29, 073116. [CrossRef]

32. Jun, Z.; Kim, C.-G. Positive solutions for a Lotka-Volterra prey-predator model with cross-diffusion of fractional type. Results Math. 2014, 65, 293-320. [CrossRef]

33. Matlob, M.A.; Towers, I.N.; Jovanoski, Z.; Irwin, A.J. Memory and mutualism in species sustainability: A time-fractional Lotka-Volterra model with harvesting. arXiv 2020, arXiv:1904.12340v2.

34. Wang, Y.; Liu, S. Fractal analysis and control of the fractional Lotka-Volterra model. Nonlinear Dyn. 2019, 95, 1457-1470. [CrossRef]

35. Kilbas, A.A.; Srivastava, H.M.; Trujillo, J.J. Theory and Applications of Fractional Differential Equations, 1st ed.; Elsevier: Amsterdam, The Netherlands, 2006; ISBN 0444518320/9780444518323.

36. Podlubny, I. Fractional Differential Equations, 1st ed.; Academic Press: San Diego, CA, USA, 1999; ISBN 558840-2.

37. Cattani, C.; Srivastava, H.M.; Yang, X.-J. (Eds.) Fractional Dynamics, 1st ed.; Springer: Berlin, Germany, 2015; ISBN 978-3-11-047209-7.

38. Baleanu, D.; Diethelm, K.; Scalas, E.; Trujillo, J.J. Fractional Calculus. Models and Numerical Methods, 2nd ed.; World Scientific: Hackensack, NJ, USA, 2016; ISBN 9813140038/978-9813140035.

39. Stamova, I.M.; Stamov, G.T. Functional and Impulsive Differential Equations of Fractional Order: Qualitative Analysis and Applications, 1st ed.; CRC Press, Taylor and Francis Group: Boca Raton, MA, USA, 2017; ISBN 9781498764834. 
40. Zhang, T.; Xiong, L. Periodic motion for impulsive fractional functional differential equations with piecewise Caputo derivative. Appl. Math. Lett. 2020, 101, 106072. [CrossRef]

41. Debbouche, A.; Baleanu, D. Controllability of fractional evolution nonlocal impulsive quasilinear delay integro-differential systems. Comput. Math. Appl. 2011, 62, 1442-1450. [CrossRef]

42. Stamov, G.; Stamova, I. Modelling and almost periodic processes in impulsive Lasota-Wazewska equations of fractional order with time-varying delays. Quaest. Math. 2017, 40, 1041-1057. [CrossRef]

43. Stamov, G.; Stamova, I., Impulsive delayed Lasota-Wazewska fractional models: Global stability of integral manifolds. Mathematics 2019, 7, 1025. [CrossRef]

44. Stamov, G.; Stamova, I. On almost periodic processes in impulsive fractional-order competitive systems. J. Math. Chem. 2018, 56, 583-596. [CrossRef]

45. Li, Y.; Chen, Y.; Podlubny, I. Mittag-Leffler stability of fractional order nonlinear dynamic systems. Automatica 2009, 45, 1965-1969. [CrossRef]

46. Li, H.; Kao, Y.G. Mittag-Leffler stability for a new coupled system of fractional-order differential equations with impulses. Appl. Math. Comput. 2019, 361, 22-31. [CrossRef]

47. Stamova, I.M. Global Mittag-Leffler stability and synchronization of impulsive fractional-order neural networks with time-varying delays. Nonlinear Dyn. 2014, 77, 1251-1260. [CrossRef]

48. Zhang, X.; Niu, P.; Ma, Y.; Wei, Y.; Li, G. Global Mittag-Leffler stability analysis of fractional-order impulsive neural networks with one-side Lipschitz condition. Neural Netw. 2017, 94, 67-75. [CrossRef]

49. Li, H.-L.; Zhang, L.; Hu, C.; Jiang, H.; Cao, J. Global Mittag-Leffler synchronization of fractional-order delayed quaternion-valued neural networks: Direct quaternion approach. Appl. Math. Comput. 2020, 373, 125020. [CrossRef]

50. Pratap, A.; Raja, R.; Rajchakit, G.; Cao, J.; Bagdasar, O. Mittag-Leffler state estimator design and synchronization analysis for fractional-order BAM neural networks with time delays. Intern. J. Adapt. Control Signal Process. 2019, 33, 855-874. [CrossRef]

51. Zhou, H.-C.; Lv, C.; Guo, B.-Z.; Chen, Y.Q. Mittag-Leffler stabilization for an unstable time-fractional anomalous diffusion equation with boundary control matched disturbance. Intern. J. Robust Nonlinear Control 2019, 29, 4384-4401. [CrossRef]

52. Ding, X.; Cao, J.; Zhao, X.; Alsaadi, F.E. Mittag-Leffler synchronization of delayed fractional-order bidirectional associative memory neural networks with discontinuous activations: State feedback control and impulsive control schemes. Proc. R. Soc. A Math. Phys. Eng. Sci. 2017, 473, 2204. [CrossRef] [PubMed]

53. Pratap, A.; Raja, R.; Sowmiya, C.; Bagdasar, O.; Cao, J.; Rajchakit, G. Robust generalized Mittag-Leffler synchronization of fractional order neural networks with discontinuous activation and impulses. Neural Netw. 2018, 103, 128-141. [CrossRef]

54. Stamova, I.; Stamov, G. Mittag-Leffler synchronization of fractional neural networks with time-varying delays and reaction-diffusion terms using impulsive and linear controllers. Neural Netw. 2017, 96, 22-32. [CrossRef] [PubMed]

55. Ballinger, G.; Liu, X. Practical stability of impulsive delay differential equations and applications to control problems. In Optimization Methods and Applications. Applied Optimization; Yang, X., Teo, K.L., Caccetta, L., Eds.; Kluwer: Dordrecht, The Netherlands, 2001; Volume 52, pp. 3-21.

56. Lakshmikantham, V.; Leela, S.; Martynyuk, A.A. Practical Stability of Nonlinear Systems; World Scientific: Teaneck, NJ, USA, 1990; ISBN 981-02-0351-9/981-02-0356-X.

57. Yoshizawa, T. Asymptotic behavior of solutions of non-autonomous system near sets. J. Math. Kyoto Univ. 1962, 1, 303-323. [CrossRef]

58. Çicek, M.; Yaker, C.; Gücen, M.B. Practical stability in terms of two measures for fractional order systems in Caputo's sense with initial time difference. J. Frankl. Inst. 2014, 351, 732-742. [CrossRef]

59. Bohner, M.; Stamova, I.; Stamov, G. Impulsive control functional differential systems of fractional order: Stability with respect to manifolds. Eur. Phys. J. Spec. Top. 2017, 226, 3591-3607. [CrossRef]

60. Stamov, G.; Stamova, I.M.; Li, X.; Gospodinova, E. Practical stability with respect to h-manifolds for impulsive control functional differential equations with variable impulsive perturbations. Mathematics 2019, 7, 656. [CrossRef]

61. Stamova, I.M.; Henderson, J. Practical stability analysis of fractional-order impulsive control systems. ISA Trans. 2016, 64, 77-85. [CrossRef]

62. Hingu, D. Asymptotic stability of strongly uninvadable sets. Ann. Oper. Res. 2020, 287, 737-749. [CrossRef] 
63. Li, Y.; Sanfelice, R.G. finite time stability of sets for hybrid dynamical systems. Automatica 2019, 100, $200-211$. [CrossRef]

64. Mironchenko, A. Uniform weak attractivity and criteria for practical global asymptotic stability. Syst. Control Lett. 2017, 105, 92-99. [CrossRef]

65. Stocker, C. Event-Based State-Feedback Control of Physically Interconnected Systems, 1st ed.; Logos: Berlin, Germany, 2014; ISBN 3832536825.

(C) 2020 by the authors. Licensee MDPI, Basel, Switzerland. This article is an open access article distributed under the terms and conditions of the Creative Commons Attribution (CC BY) license (http:/ / creativecommons.org/licenses/by/4.0/). 